\title{
A Multispacecraft Analysis of a Small-Scale Transient Entrained by Solar Wind Streams
}

\author{
A.P. Rouillard · N.P. Savani · J.A. Davies · B. Lavraud • R.J. Forsyth • S.K. Morley • \\ A. Opitz • N.R. Sheeley · L.F. Burlaga · J.-A. Sauvaud • K.D.C. Simunac • \\ J.G. Luhmann • A.B. Galvin • S.R. Crothers • C.J. Davis • R.A. Harrison • \\ M. Lockwood · C.J. Eyles · D. Bewsher · D.S. Brown
}

Received: 7 December 2008 / Accepted: 21 February 2009 / Published online: 19 March 2009

(c) The Author(s) 2009. This article is published with open access at Springerlink.com

STEREO Science Results at Solar Minimum

Guest Editors: Eric R. Christian, Michael L. Kaiser, Therese A. Kucera, O.C. St. Cyr

A.P. Rouillard $(\bowtie) \cdot$ M. Lockwood

Space Environment Physics Group, School of Physics and Astronomy, University of Southampton, Southampton, SO17 1BJ, UK

e-mail: alexisrouillard@yahoo.co.uk

A.P. Rouillard · J.A. Davies · S.R. Crothers · C.J. Davis · R.A. Harrison · M. Lockwood · C.J. Eyles · D. Bewsher

Space Science and Technology Department, Rutherford Appleton Laboratory, Chilton, Oxfordshire, OX11 0QX, UK

N.P. Savani · R.J. Forsyth

Space and Atmospheric Physics, Blackett Laboratory, Imperial College London, London, SW7 2BW, UK

B. Lavraud · A. Opitz · J.-A. Sauvaud

Centre d'Etude Spatiale des Rayonnements, Université de Toulouse (UPS), Toulouse, France

B. Lavraud · A. Opitz · J.-A. Sauvaud

Centre National de la Recherche Scientifique, UMR 5187, Toulouse, France

S.K. Morley

Centre for Space Physics, University of Newcastle, Newcastle, NSW 2308, Australia

N.R. Sheeley

Space Science Division, Naval Research Laboratory, Washington, DC 20375-5352, USA

L.F. Burlaga

Goddard Space Flight Center, NASA/GSFC, Code 673, Greenbelt, MD 20771, USA

K.D.C. Simunac · A.B. Galvin

Institute for the Study of Earth Oceans and Space, University of New Hampshire, Durham, NH 03824, USA 
Abstract The images taken by the Heliospheric Imagers (HIs), part of the SECCHI imaging package onboard the pair of STEREO spacecraft, provide information on the radial and latitudinal evolution of the plasma compressed inside corotating interaction regions (CIRs). A plasma density wave imaged by the HI instrument onboard STEREO-B was found to propagate towards STEREO-A, enabling a comparison between simultaneous remotesensing and in situ observations of its structure to be performed. In situ measurements made by STEREO-A show that the plasma density wave is associated with the passage of a CIR. The magnetic field compressed after the CIR stream interface (SI) is found to have a planar distribution. Minimum variance analysis of the magnetic field vectors shows that the SI is inclined at $54^{\circ}$ to the orbital plane of the STEREO-A spacecraft. This inclination of the CIR SI is comparable to the inclination of the associated plasma density wave observed by HI. A small-scale magnetic cloud with a flux rope topology and radial extent of $0.08 \mathrm{AU}$ is also embedded prior to the SI. The pitch-angle distribution of suprathermal electrons measured by the STEREO-A SWEA instrument shows that an open magnetic field topology in the cloud replaced the heliospheric current sheet locally. These observations confirm that HI observes CIRs in difference images when a small-scale transient is caught up in the compression region.

Keywords Sun: magnetic field $\cdot$ Sun: corotating interaction regions $\cdot$ Interplanetary medium: coronal mass ejection

\section{Introduction}

The heliospheric plasma is formed by the continuous supersonic outflow of the solar wind (Parker, 1958) and the continual emergence of small- and large-scale transients (Crooker et al., 2004; Hudson, Bougeret, and Burkepile, 2006). Solar wind streams originate from lowdensity, magnetically open regions of the Sun's corona, known as coronal holes, where solar plasma continuously escapes along magnetic field lines which link the photospheric regions to the interplanetary medium. A single coronal hole can be observed for several successive solar rotations (Insley, Moore, and Harrison, 1995) and, thus, coronal holes are considered long-lived and stable structures (Wang, Hawley, and Sheeley, 1996). Therefore the speed of the plasma outflowing from these regions can also be considered to be relatively stable (Luhmann et al., 2002). The background solar wind flow speed measured near the Earth can, however, vary from low values $\left(\sim 300-400 \mathrm{~km} \mathrm{~s}^{-1}\right)$ to high ones $\left(\sim 600-700 \mathrm{~km} \mathrm{~s}^{-1}\right)$ in a matter of days. This rapid variation is the result of solar rotation bringing sources of fast and slow solar wind at adjacent longitudes into radial alignment, allowing, on occasion, fast solar wind to interact with slow solar wind during its outward expansion. In such corotating interaction regions (CIRs) the ambient plasma is compressed (Pizzo and Gosling, 1994). The evolution of CIRs is reasonably well understood following intensive analytical (Lee, 2002)

J.G. Luhmann

Space Science Laboratory, University of California, Berkeley, CA 94720, USA

C.J. Eyles

Grupo de Astronomia y Ciencias del Espacio, Universidad de Valencia, Valencia, Spain

D. Bewsher · D.S. Brown

Institute of Mathematical and Physical Sciences, University of Wales Aberystwyth, Aberystwyth, SY23 3BZ, UK 
and numerical work (Pizzo and Gosling, 1994). CIRs are the most common high-density structures observed in situ during the years of solar minimum (Gazis, 1996).

The transient heliospheric plasma is composed of a large variety of magnetohydrodynamic (MHD) structures, which range from high plasma beta structures (Crooker et al., 2004) to the more extensive coronal mass ejections (CMEs) and their associated flux ropes (Hudson, Bougeret, and Burkepile, 2006). The interaction between the background and the transient parts of the solar wind has been investigated for many years, mainly using spacecraft located at $1 \mathrm{AU}$ and beyond (Burlaga, Hewish, and Behannon, 1991). In particular, the entrainment of CMEs by solar wind streams was studied in great detail using observations from the Voyager and Pioneer spacecraft (e.g., Burlaga et al., 1985). The limited fields of view of coronagraphs mean that - prior to the launch of the Coriolis spacecraft with its Solar Mass Ejection Imager (SMEI) and the launch of the Solar TErrestrial Relations Observatory (STEREO) - most studies had considered the coupling between the background solar wind and solar transients between the Sun and 1 AU by analysing in situ observations of the solar wind (Burlaga, Hewish, and Behannon, 1991). Yet a detailed assessment of the influence of solar wind transients on the properties of the solar wind at $1 \mathrm{AU}$ and beyond has important consequences for our understanding of long-term changes in solar wind - magnetosphere coupling (Fenrich and Luhmann, 1998; Lavraud et al., 2006) and the modulation of galactic cosmic rays (Burlaga et al., 2003). Previous studies have shown, for instance, that CMEs that interact significantly with the slow background solar wind are more geoeffective than those that propagate in a uniform fast solar wind (Burlaga, Behannon, and Klein, 1987).

Initial observations made by the Heliospheric Imagers (HIs) (Eyles et al., 2009), part of the SECCHI imaging package (Howard et al., 2008a) onboard the STEREO spacecraft, suggested that HI could be used to observe CIRs (Rouillard et al., 2008; Sheeley et al., 2008a, 2008b). In particular, the comparison of simultaneous white-light images and in situ observations of the solar wind showed that the large plasma density waves observed by $\mathrm{HI}$ are associated with the passage of CIRs through the inner and outer HI fields of view (termed HI-1 and HI-2, respectively). Rouillard et al. (2008) and Sheeley et al. (2008a, 2008b) noted that these CIR-associated waves appear in a series, with a separation between each wave of several hours. This effect was attributed to the continual release of small-scale transients in the slow solar wind, which are subsequently compressed in CIRs, effectively forcing additional longitudinal structure inside the dense CIR spiral arm. This interpretation is examined in more detail in the present paper.

\section{Instruments}

The two spacecraft of the NASA STEREO mission, STEREO-A and STEREO-B, carry near-identical instrumentation that includes the SECCHI package comprising an Extreme Ultraviolet Imager (EUVI), two coronagraphs (COR-1 and COR-2) and the Heliospheric Imagers (HIs). The HI instrument on each STEREO spacecraft consists of two white-light, wide-field imagers, HI-1 and HI-2, which, like coronagraphs, detect sunlight scattered from coronal electrons by Thomson scattering (Vourlidas and Howard, 2006). The inner HI-1 cameras have a $20^{\circ}$ square field of view (fov), with boresites aligned at $14^{\circ}$ elongation in the elliptic plane. The boresites of the outer (circular fov of diameter $70^{\circ}$ ) HI- 2 cameras are aligned at $53.3^{\circ}$ elongation in the ecliptic. Figure 1a presents a view of the ecliptic plane from above on 19 July 2007 showing the relative positions of STEREO-A (A), STEREO-B (B) and the Earth (E). The elongation extents in that plane of the fovs of HI-1 and HI-2 on 
Figure 1 (a) View of the ecliptic plane from above showing the location of the Earth (E), STEREO-A (A) and STEREO-B (B) on 19 July 2007. The limits, in this plane, of the fov of HI-1B (red) and HI-2B (blue) are also marked. (b) The extents of the fov in elongation and elevation $(\epsilon)$ of HI-1B (red) and HI-2B (blue); the position of Ulysses on 24 July 2007 is also shown (U).

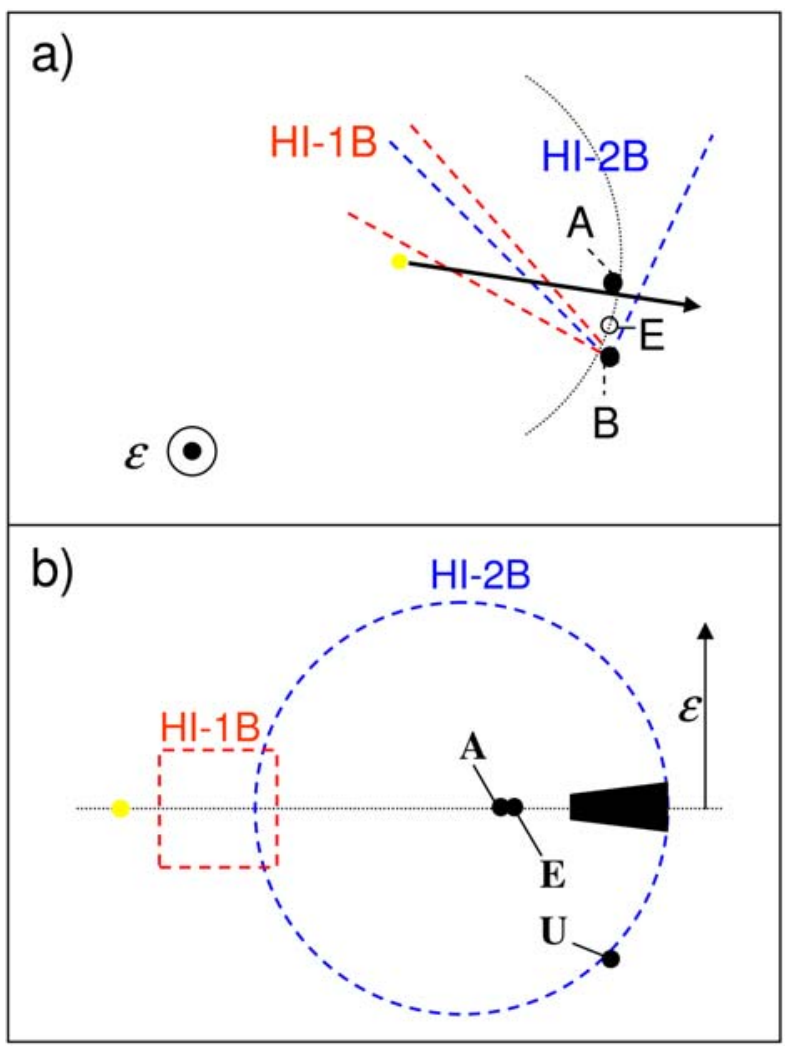

STEREO-B (termed HI-1B and HI-2B, respectively) are marked in red and blue. The extent of the fields of view of HI-1B and HI-2B out of the ecliptic plane (in elevation, $\epsilon$ ) are shown in Figure 1b. The Earth and STEREO-A were in the HI-2B fov during this early part of the mission, so that the solar wind can be tracked continuously from the Sun to either. The location of the Ulysses spacecraft (U) on 24 July 2007 is also shown; this corresponds to the date that Ulysses recorded the passage of the CIR analysed in this paper.

In addition to the SECCHI imaging suite, each of the STEREO spacecraft also carries a comprehensive suite of in situ instrumentation, including the Plasma and Suprathermal Ion Composition (PLASTIC) (Galvin et al., 2008) and the In Situ Measurements of Particles and CME Transients (IMPACT) packages (Luhmann et al., 2008). Magnetic field measurements from the magnetometer (MAG) (Acuña et al., 2008) and suprathermal electron observations taken by the Solar Wind Electron Analyser (SWEA) (Sauvaud et al., 2008), two components of the IMPACT package, are used in the present study. Near-Earth solar wind observations from the Solar Wind Electron, Proton, Alpha Monitor (SWEPAM) investigation (McComas et al., 1998) onboard the Advanced Composition Explorer (ACE) are also used. As we shall see, the STEREO-A/B and ACE spacecraft form an ideal constellation of spacecraft to study solar wind structures near $1 \mathrm{AU}$. 


\section{Observation of a Plasma Density Wave in July 2007}

The light scattered off the electrons compressed in the spiral arm of a CIR should influence HI-1/2 images during a twelve-day period (Rouillard et al., 2008). This period corresponds roughly to the time required for the source region of the CIR to corotate by $90^{\circ}$ plus a five-day transit time for the compressed plasma to travel a distance of $\sim 1$ AU near the outer edge of the outer camera. This slow variation can be simulated by using ray tracing models which approximate the effect of Thomson scattering (Howard et al., 2008b). During the interval presented in this paper, images were taken every 40 minutes by HI-1B and every two hours by HI-2B. Running difference images obtained from consecutive images taken over 40-minute to 2-hour intervals should therefore diminish the slowly varying CIR signal and only reveal the variations of the CIR spiral forced by the presence of smallscale transients trapped inside the CIR. This variations of the CIR will propagate radially outwards with the solar wind plasma (i.e., along a specific solar longitude). A transient moving radially outwards at $300 \mathrm{~km} \mathrm{~s}^{-1}$ can cross $10-20 \mathrm{HI}-1$ pixels between successive 40-minute images and $8-16 \mathrm{HI}-2$ pixels between successive 2-hour intervals depending on the angle of propagation; consecutive images of such a transient will therefore be different and running difference images will consequently track its outward motion. We present a detailed study of one such CIR variation using a combination of white-light images and in situ measurements taken during July 2007. This paper aims to answer two questions: (1) Can the geometry of the large-scale CIR variations seen by HI be interpreted in terms of the in situ observations of this CIR? (2) Is there a small-scale transient embedded inside this CIR-associated wave as the current theory suggests?

Figure 2 presents a composite of HI-1B and HI-2B difference images showing a largescale outflowing density increase (black/white region) from 21:30 UT on 19 July 2007. Note that the running difference technique minimises the contribution of the stable F-corona and is very useful for highlighting faint propagating features, as demonstrated by Sheeley et al. $(1999,2008 \mathrm{a}, 2008 \mathrm{~b})$. The feature corresponds to a single ripple or wave of density variation propagating outwards from the Sun (from left to right on this plot). Sheeley et al. (2008b) showed that this density variation is consistent with plasma being compressed by a CIR (observed in situ). As stated in the introduction, CIRs are the result of fast solar wind interacting with slow solar wind propagating upstream along the same solar radial. The large-scale density increase seen in Figure 2 has a clear inclination. This spatial distribution of the plasma, if CIR-associated, should have important consequences for the magnetohydrodynamic properties of the CIR observed in situ (Lee, 2002); we investigate this in Section 5 after having determined the trajectory of the transient (in the next section).

\section{Determining the Trajectory of the HI-2B Waves}

The elongation, $\alpha$, of a plasma parcel, $\mathrm{T}$, in the solar wind as observed by the STEREO-B spacecraft is defined as the Sun - STEREO-B - T angle, being zero at Sun centre. This angle is marked in the schematic shown in Figure 3. The angular separation between the SunSTEREO-B line and the direction of propagation of T (i.e., the transient's path) is marked $\beta$ in the figure. Figure 4 presents a stack plot created by extracting lines at a constant position angle (PA) of $265^{\circ}$ from solar north from a series of HI-1/2B composite difference images and plotting them vertically as a function of $\alpha$. This PA line is shown in Figure 2 and was chosen to avoid the intense signature of the Earth (and its image ghost) on the central rows of the HI-2B image (aligned with the solar ecliptic). Plots such as that presented in Figure 4 are 
Figure 2 A composite of HI-1B and HI-2B difference images from 19 July 2007 during the passage of the plasma density wave. These images are shown in helioprojective Cartesian coordinates. Lines of constant elongation are overlaid as well as the line of constant PA $\left(=265^{\circ}\right)$ used to create the $\mathrm{J}$ map. The location of STEREO-A (A) is shown in the HI-2 difference image. Earth is located very close to STEREO-A in the HI-2B fov. $\mathrm{S}$ marks the location of the Sun.
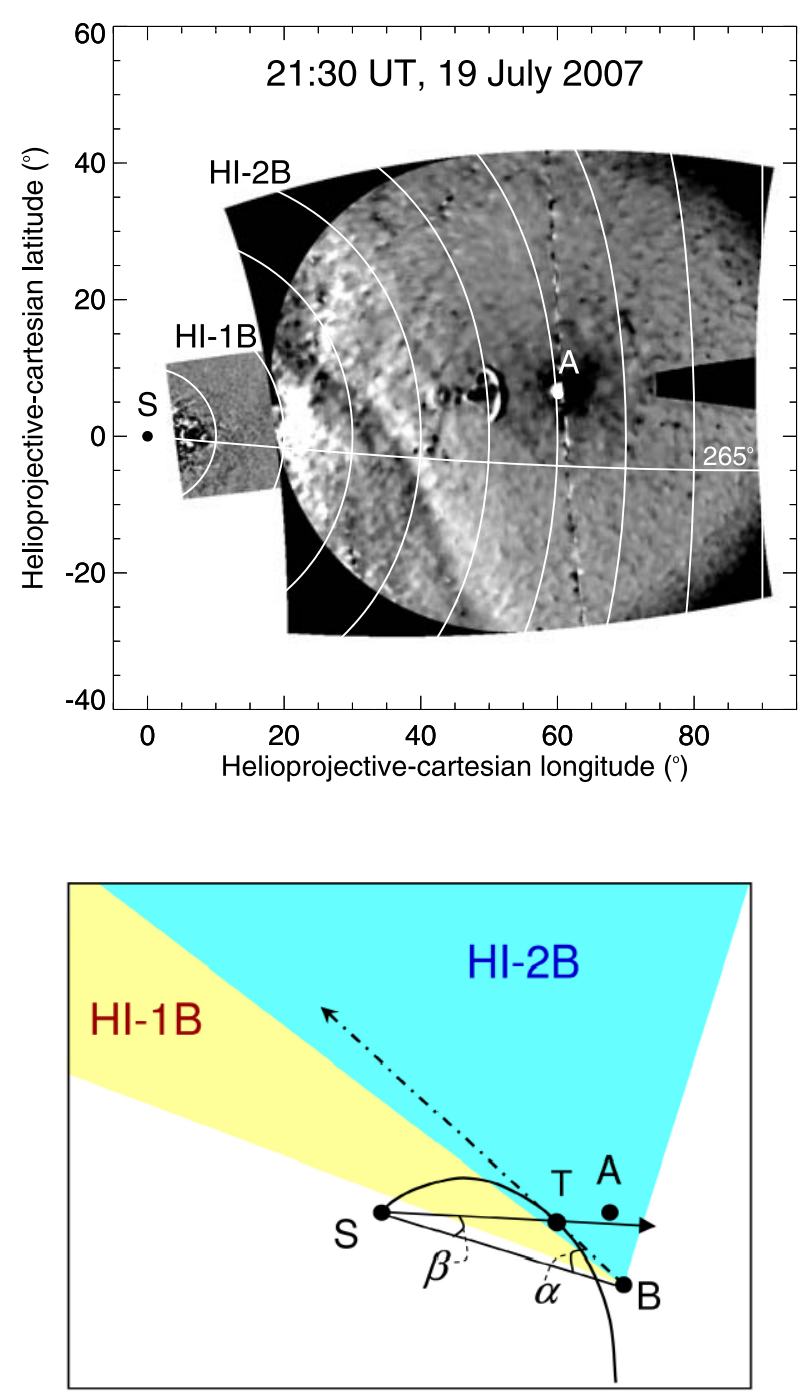

Figure 3 Schematic of the small-scale transient (T) propagating inside the spiral arm of a CIR in the intersection of the HI-1B (yellow region) and HI-2B (blue region) fields of view in the equatorial plane. The angles $\alpha$ and $\beta$ referred to in the paper are shown on this figure together with the position of the Sun (S), STEREO-A (A) and STEREO-B (B).

called $\mathrm{J}$ maps because of the imprint of the acceleration phase of a solar transient (Sheeley et al., 1999). Rouillard et al. (2008) and Sheeley et al. (2008a, 2008b) showed that the apparent acceleration/deceleration at the elongations covered by HI $\mathbf{J}$ maps is mostly an effect of projection geometry. Plasma parcels propagating towards (away from) the observer will have a gradually accelerating (decelerating) elongation variation. The authors showed that when plasma elements are released by a single corotating source region, tracks associated with these elements will appear to converge and diverge in J maps constructed from STEREOA and STEREO-B images, respectively. Two clear tracks can be seen in Figure 4 (seen at elongation $\alpha=40^{\circ}$ on 20 July and on 24 July); these result from the presence of two plasma parcels emerging from the same corotating interaction region. The wave in Figure 2, which corresponds to the first distinct track in the $\mathrm{J}$ plot, appears to accelerate during its outward propagation. 


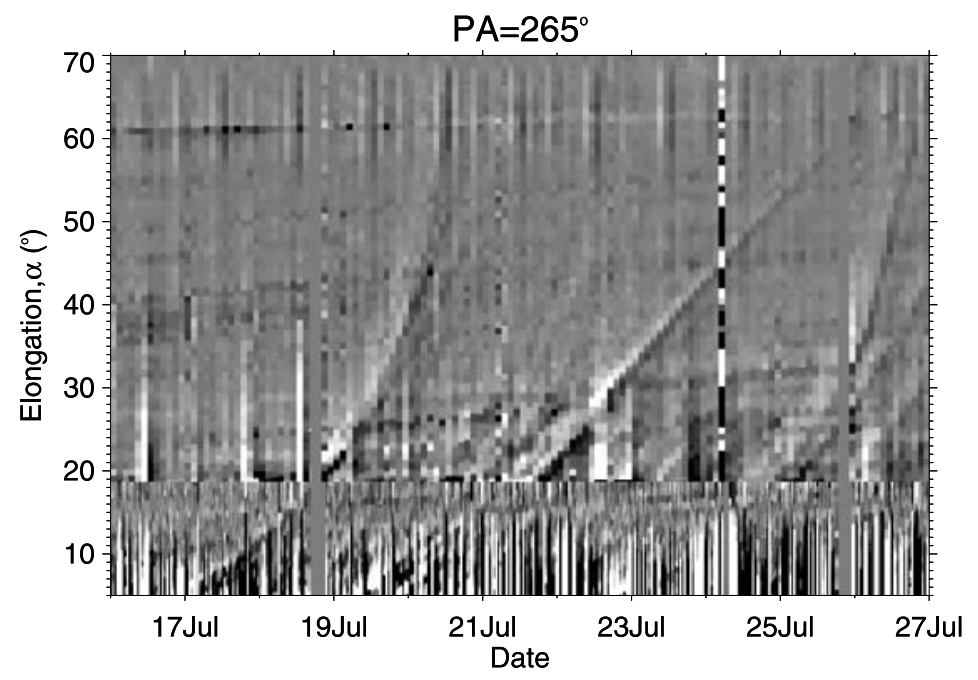

Figure 4 A J map extracted along $\mathrm{PA}=265^{\circ}$ for the period 16 to $27 \mathrm{July} 2007$, derived from HI images from STEREO-B. The first clear track on this map is the signature of the wave shown in Figure 2; a pronounced gradual (apparent) acceleration can be observed. The second clear track (apparently decelerating) is also related to the same corotating interaction region passing in the field of view a few days later but at a greater $\beta$ angle.

The apparent acceleration revealed by the J-mapping technique can be used to predict the trajectory of the associated solar wind transient if one assumes that the transient propagates radially outwards and at a constant speed. The elongation variation, $\alpha(t)$, of a solar wind transient depends upon its radial speed, $V_{\mathrm{r}}$, and the angle $\beta$ (Rouillard et al., 2008; Sheeley et al., 2008a, 2008b) as

$$
\alpha(t)=\operatorname{atan}\left[\frac{V_{\mathrm{r}} t \sin \beta}{r_{\mathrm{B}}(t)-V_{\mathrm{r}} t \cos \beta}\right],
$$

where $r_{\mathrm{B}}$ is the radial distance of STEREO-B. Best-fit values for $\beta$ and $V_{\mathrm{r}}$ can be obtained from the $\alpha$ elongation variation recorded by HI (Rouillard et al., 2008; Sheeley et al., 2008a, $2008 \mathrm{~b}$ ). The value of $\beta$ corresponds to the solar longitude difference between the spacecraft and the outflowing transient when the fitting is applied to a $\mathrm{J}$ map extracted along the equatorial plane. In the case of a $\mathrm{J}$ map taken at a PA greater or lower than that of the solar equatorial plane (i.e., not $0^{\circ}$ or $180^{\circ}$ ), a correction must be applied to the $\beta$ value obtained from Equation (1) to obtain the heliocentric longitude difference $\left(\psi=\psi_{\mathrm{B}}-\psi_{\mathrm{T}}\right)$ between the longitude of the solar radial along which the transient is propagating $\left(\psi_{\mathrm{T}}\right)$ and the longitude of STEREO-B $\left(\psi_{\mathrm{B}}\right)$. The heliocentric latitude and longitude of the transient tracked along $\mathrm{PA}=265^{\circ}$ and its source region can be extracted by using this method provided the tilt of the Sun relative to STEREO-B is also accounted for (the so-called $B_{0}$ tilt) (Sheeley et al., 2009). A best fit of the apparent acceleration of the outflowing solar wind parcel imaged in Figure 2 was found for $\beta=17^{\circ}$ relative to the Sun-STEREO-B line and a speed of $340 \mathrm{~km} \mathrm{~s}^{-1}$. This angle of propagation corresponds to a longitude difference of $\psi=16^{\circ}$ and is shown in Figure 3 by a solid arrow through $S$ and $T$. The longitudinal separation of STEREO-A and STEREO-B was $19^{\circ}$ at the time. The section of the CIR imaged by HI on STEREO-B in Figure 2 was therefore propagating towards the STEREO-A spacecraft. 


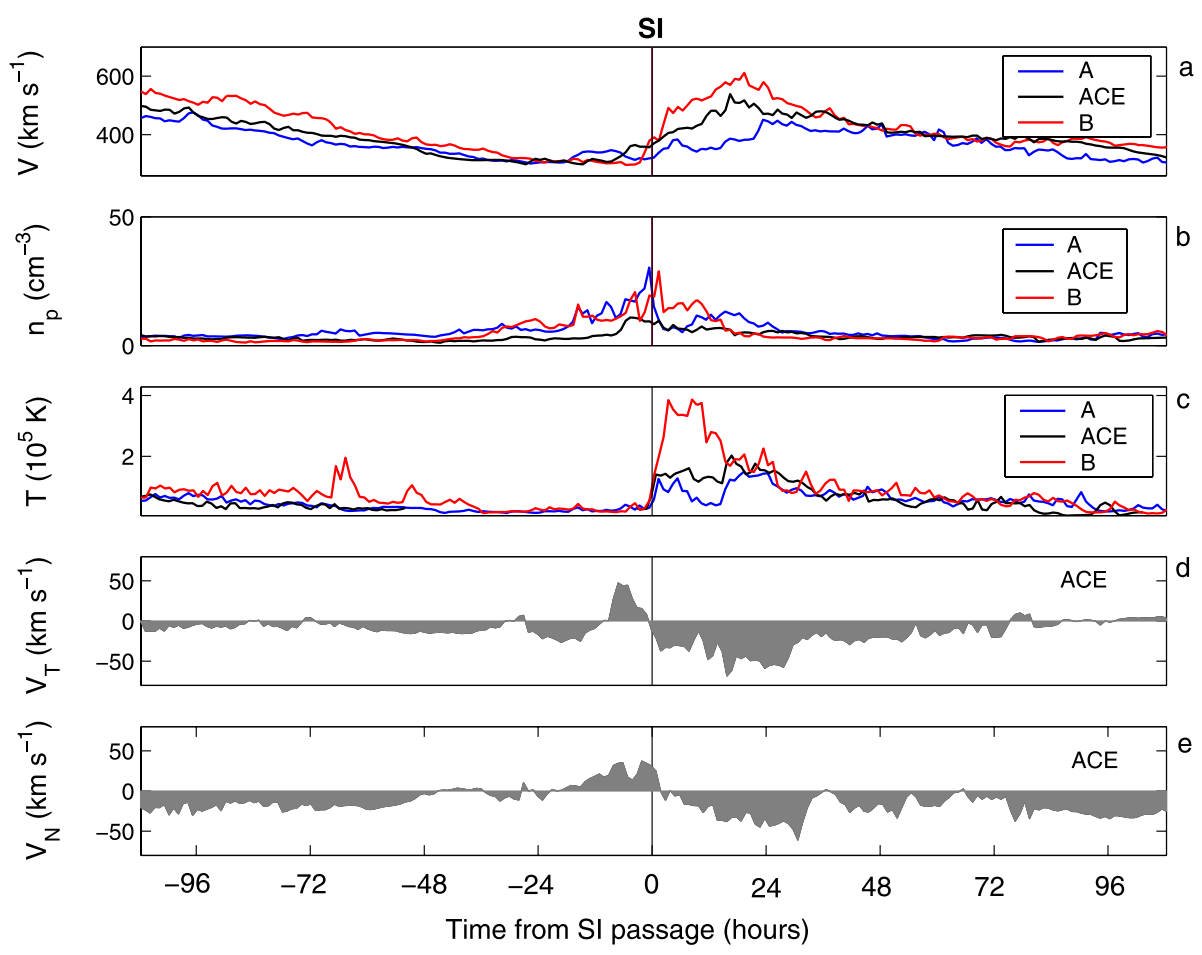

Figure 5 A superposed epoch analysis of the proton speed (a), density (b) and temperature (c) from PLASTIC on STEREO-A and -B and ACE/SWEPAM, and ACE/SWEPAM measurements of the azimuthal and latitudinal deflection of the solar wind flows ( $d$ and e) of the same CIR. Time 0 marks the passage of the SI defined here by a sudden increase in proton temperature.

The plasma parcel first visible in the J plot on 17 July 2008 remains visible to the limit of the HI-2B fov. As noted by Sheeley et al. (2008b) the presence of STEREO-A and the Earth in the HI-2B fov makes this feature ideally suited for a combined in situ and white-light analysis. Moreover, STEREO-A lies close to the Thomson sphere of STEREO-B (Vourlidas and Howard, 2006) at this time, which allows a detailed observation of the parcel until its impact at spacecraft A.

\section{Comparison between HI Observations and In Situ Data}

Proton speed, density and temperature observations from the PLASTIC instruments on STEREO-A and STEREO-B and the SWEPAM instrument on ACE are shown in Figures 5a, $5 \mathrm{~b}$ and $5 \mathrm{c}$, respectively. The tangential $(\mathrm{T})$ and normal $(\mathrm{N})$ components of the solar wind speed measured by ACE in RTN coordinates are shown in Figures 5d and 5e, respectively. The CIR associated with the density wave seen in HI-2B sweeps over all three spacecraft. However, from the trajectory estimate of the density wave $\left(\psi=16^{\circ}\right)$, only STEREO-A was hit by that section of the CIR imaged by HI on STEREO-B. The (tenuous) fast and (dense) slow solar wind streams are usually separated by a stream interface (SI) (Burlaga, 1974; Gosling et al., 1978). The SI was identified by its sudden increase in proton temperature and the drop in density, associated with the change from dense and cold slow solar wind to the 
more tenuous and hot fast solar wind. The three datasets shown in Figure 5 are currently superposed by setting the time of the SI crossing as zero epoch. The solar wind data at all three spacecraft are consistent with the successive passage of a CIR at STEREO-B, ACE and then STEREO-A; in particular, an increase in solar wind speed is observed in association with an increase in density inside the compression region. Components of the solar wind velocity were only available from ACE SWEPAM. Figure 5d reveals the expected East/West deflection of the slow/fast solar wind around the SI (Lee, 2002).

The solar wind speed profile is steeper with faster solar wind at STEREO-B than at ACE or STEREO-A. The difference in the observed solar wind speed measurements is related either to dynamic changes in the coronal hole morphology (and therefore the speed of the wind escaping the coronal hole) or to the different latitudinal positions of the three spacecraft. These two hypotheses can be evaluated by using images of the lower corona. Figure 6 shows an EUV image of the corona taken at $195 \AA$ by the EUV imager on STEREO-B at the time of launch of the plasma structure shown in Figure 2. The source region of the density structure tracked along $\mathrm{PA}=265^{\circ}$ was identified using the $\mathrm{J}$ map (Figure 4), assuming a constant-speed back-mapping of the plasma parcel to the source surface $\left(2.5 R_{\odot}\right)$; this source region is marked by a red dot on the image and is located on the western boundary of a significant equatorial coronal hole, which is mainly located south of the solar equator. The size and morphology of this coronal hole did not detectably evolve during the analysis of the corona presented in this paper. Helios observations (Schwenn and Marsch, 1990) showed that a latitudinal separation of only $1^{\circ}$ between two spacecraft can lead to significant observational differences as they observe different latitudinal slices of the same coronal hole. STEREO-B and ACE were located $2.2^{\circ}$ and $1^{\circ}$ south of STEREO-A, respectively, and Figure 6 shows that they therefore would have been in a more favourable position than STEREO-A to observe the fast flow out of the coronal hole. The ordering of the in situ measurements, with an increasingly fast solar wind speed and higher temperatures further south, suggests that the latitudinal position of the spacecraft affected the in situ observations significantly. The coronal hole seen in the EUV image is therefore associated with the CIR observed in situ at STEREO-A/B and ACE and by inference also associated with the formation of the density wave seen in Figure 2.

The density enhancement in a CIR is usually located before the SI (Burlaga, 1974; Gosling et al., 1978). As a further test to determine whether the wave seen in HI-2B was partly CIR associated, the SI detected in situ by STEREO-A was mapped ballistically to time 21:30 UT on 19 July 2007 (the time of Figure 2), along a longitude of $\psi=16^{\circ}$ relative to STEREO-B. The speed of the SI was assumed to be constant throughout the time of the mapping. The result of this mapping is shown in Figure 7 as a single dot labelled $A_{\mathrm{SI}}$. As expected, the SI is located at the rear of the plasma density wave, providing more confidence that the wave seen in HI on STEREO-B is partially CIR related.

We note that the $\mathrm{N}$ component of the solar wind speed measured by ACE and plotted in Figure 5e indicates a southern deflection of the fast flow and a northern deflection of the slow flow. These deflections would be expected from the inclination of the SI, if the SI is found to be located on the sunward flank of the plasma density wave observed in HI (Figure 7) (see Lee, 2002, for a discussion of this effect).

\section{In Situ Observations of the Magnetic Field}

\subsection{Comparing STEREO-A and STEREO-B}

An increase in solar wind speed occurs during the passage of the CIR over STEREO-B between 19 and 21 July 2007 (Figure 8e). The onset of this speed increase is associated with 
Figure 6 An EUVI image taken on 16 July 2007. These data were obtained by ballistically back-mapping the outflowing structure seen in $\mathrm{HI}$ on STEREO-B (Figure 2) to the lower corona. The source region of this feature, on the solar radial presented in the $\mathrm{J}$ map, is shown as a red dot located on the western boundary of an equatorial coronal hole. The polarity of the magnetic field lines of the three major coronal holes observed during this period are also shown.
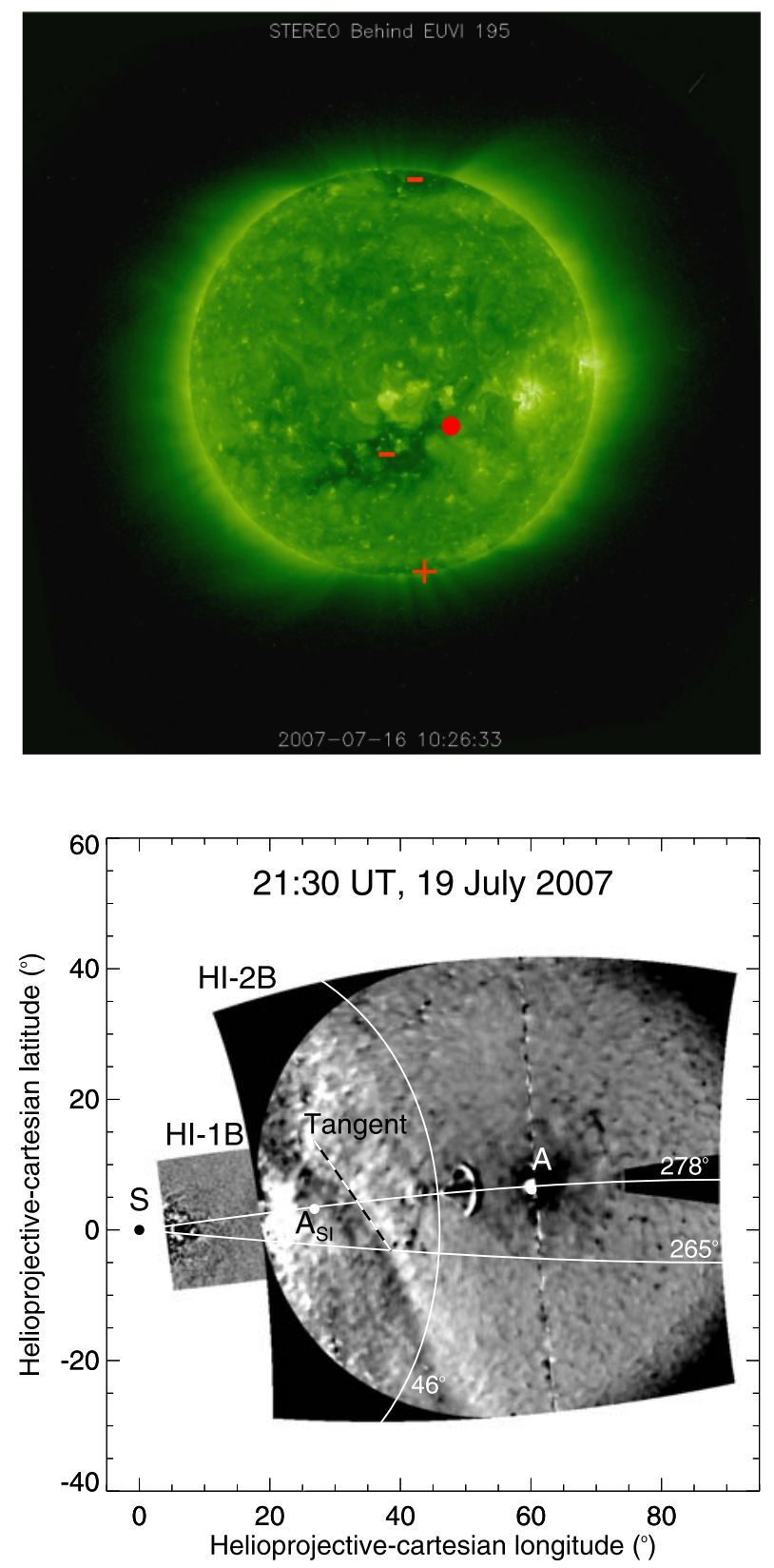

Figure 7 An annotated version of the composite image shown in Figure 2. The position of the SI predicted by ballistically back-mapping its position determined from the in situ measurements taken by STEREO-A is shown as the white dot labelled $A_{\text {SI }}$. The orientation of the SI derived from STEREO-A in situ observations is plotted as a black and white dashed line. A white line is plotted at a constant elongation of $46^{\circ}$.

an increase in plasma density in Figure 8f and magnetic field strength (Figure 8b). Solar wind electrons can be typically classified by three different populations. The main part is composed of the thermal core electrons representing $95 \%$ of the total electron density. The remaining 5\% represent the suprathermal non-Maxwellian halo, distributed in all directions of the velocity space and the strahl. This last component is mostly aligned in the direction parallel to the interplanetary magnetic field and largely moving away from the Sun. The 


\section{STEREO B}

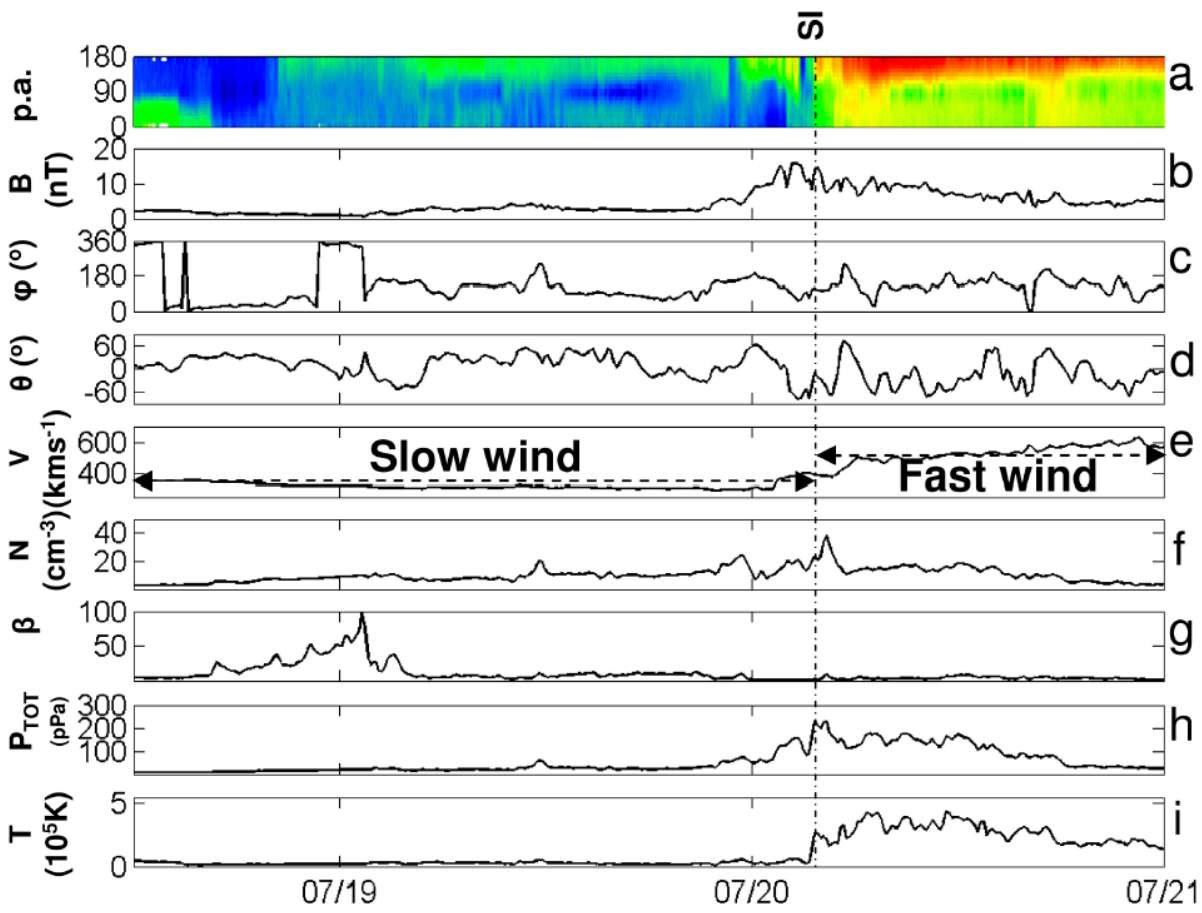

Figure 8 PLASTIC and IMPACT observations of the CIR observed in situ at STEREO-B. A pitch-angle spectrogram of $264 \mathrm{eV}$ electrons measured by PLASTIC/SWEA is presented in panel a, and the magnitude, azimuth and elevation of the magnetic field evaluated from RTN coordinates are shown in panels b, c and d, respectively. Remaining panels show IMPACT observations of solar wind proton speed (e), density (f), ion plasma beta $(\mathrm{g})$, total pressure (h) and temperature (i). The SI passage is indicated by the time of sudden proton temperature increase and density decrease.

presence of $180^{\circ}$ pitch angle suprathermal electron strahl (for $\sim 264 \mathrm{eV}$ electrons; Figure 8a) confirms that the polarity of the magnetic field (magnetic field azimuth of $\phi=135^{\circ}$ ) does not change during the CIR passage. The Heliospheric Current Sheet (HCS) is crossed by the spacecraft at 12 UT on 18 July 2007, significantly before the CIR passage.

STEREO-A observes very different solar wind conditions during the same CIR passage a few hours later. In particular, the CIR passage is associated with a clear reversal of the polarity of magnetic field lines (Figure 9c). This reversal is confirmed by a change of suprathermal electron pitch angle from $0^{\circ}$ to $180^{\circ}$ at 13 UT on 20 July 2007 (Figure 9a). The speed profile consists of three step increases. The first, around $10 \mathrm{UT}$ on $20 \mathrm{July}$, is not marked by a rise in solar wind temperature and a rather high density (Figure 9f and Figure 9i), suggesting that this plasma flow is not associated with fast solar wind. Plasma beta (Figure $9 \mathrm{~g}$ ) decreases substantially between 12 and 24 UT on this day. The passage of this low-beta structure is correlated with a smooth rotation in the magnetic field direction (Figure 9c and 9d). Such smooth rotations are characteristic of the passage of flux ropes; a flux rope with a low temperature and low plasma beta satisfies the conditions set by Burlaga et al. (1981) to classify it as a magnetic cloud. 


\section{STEREO A}

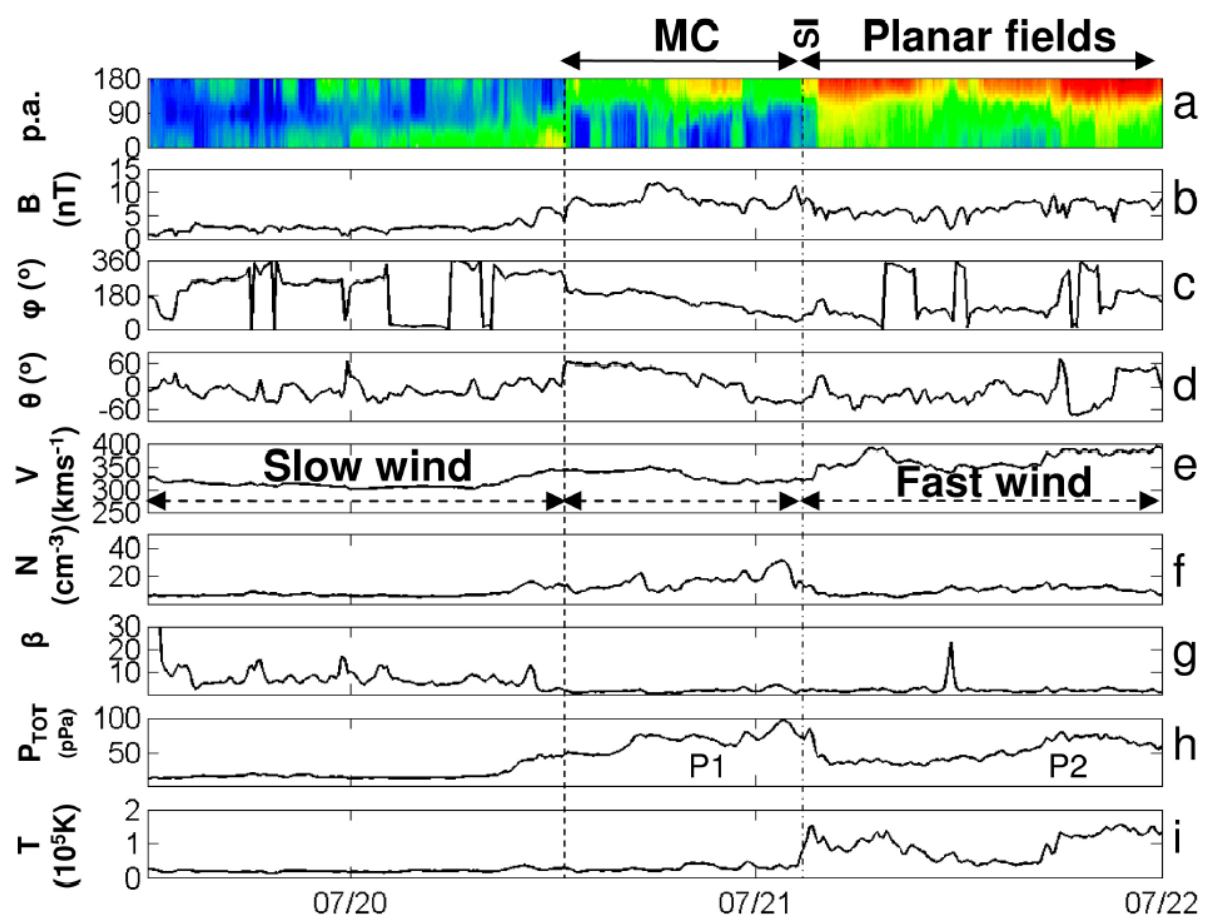

Figure 9 STEREO-A in situ observations of the CIR in the same format as Figure 8. The passage of the magnetic cloud (MC) and the planar fields are also shown.

Although not shown here, the MAG and SWEPAM instruments onboard the ACE spacecraft observed similar CIR profiles to those seen at STEREO-B. The magnetic field strength peaks at the stream interface as expected but has a second peak ahead of the interface not associated with a clear rotation of the magnetic field. This second peak could be related to the effect of the small-scale transient, which, although passing nearer the STEREO-A spacecraft, could have forced some compression of the magnetic field along its earthward edge. A shock, or a structure evolving towards a shock, may have been driven by the corotating stream interacting with the earthward edge of the magnetic cloud.

\subsection{Magnetic Field Topology inside the Cloud}

The strahl orientation reverses during the passage of the magnetic cloud as observed in situ by STEREO-A (Figure 9a), suggesting that the magnetic cloud has replaced the HCS locally. Moreover, the suprathermal electrons are not counter-streaming but have a $180^{\circ}$ pitch angle, suggesting that the magnetic field lines forming the flux rope are open. The lack of counterstreaming electrons suggests that only one end of the magnetic field lines which form the flux rope are rooted at the photospheric level (i.e., the magnetic field lines are open). The magnetic field lines of this rope have an inward polarity, which is the same polarity of the equatorial coronal hole (Figure 6). 


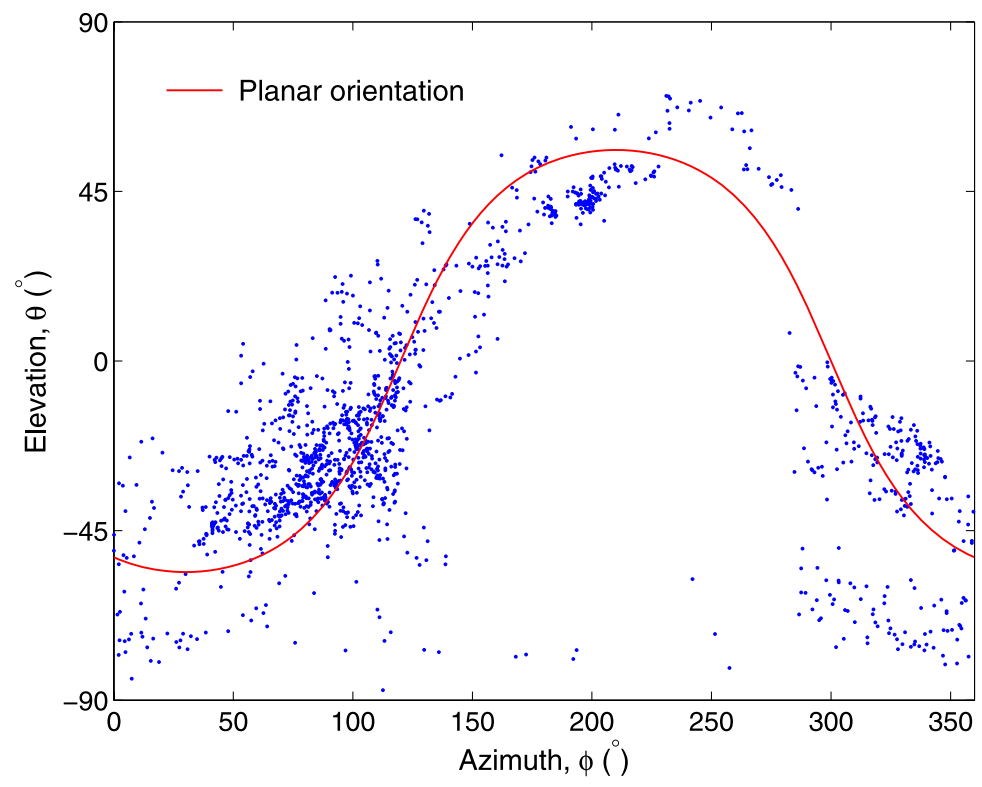

Figure 10 A plot of the elevation $(\theta)$ of the magnetic field measured at STEREO-A as a function of the azimuth $(\phi)$ for the period from 03:00 UT on 21 July to 04:00 UT on 22 July 2007. The planar orientation derived by minimum variance analysis is plotted as a thick red line.

A constant-alpha force-free-field fit of the magnetic field inside the magnetic cloud (MC) was used to determine the direction of the local orientation of the flux-rope axis; the fitting technique is the same as that used by Rees and Forsyth (2004). The flux-rope fit is found to have left-handed chirality, and the MC axis orientation in RTN coordinates is found to be $(-0.68,0.73,0.01)$. This orientation corresponds to a cloud axis parallel to the RT plane (horizontal flux rope) and aligned close to the nominal Parker spiral field. We therefore find that the STEREO-A spacecraft passed through a "mini" magnetic cloud embedded inside the CIR. This MC, which was not present at the location of STEREO-B (or ACE), is oriented along the Parker spiral field and has locally "replaced" the HCS. We further use these findings in the final discussion.

\section{Magnetic Field Orientation around the SI}

STEREO-A observations show that the solar wind speed and temperature increase on arrival of the SI; these increases are associated with a first pressure wave P1 (Figure 9h). A second increase in speed occurs around 15 UT on 21 July, marking the arrival of a second pressure wave (P2). Compression regions developed ahead of and at the rear of the main SI. The first increase in plasma density coincident with the MC/CIR system is much greater than the second purely CIR-associated increase, explaining why HI saw a single large-scale plasma density wave. The SI and post-SI periods were used to determine the orientation of the solar wind magnetic field compressed in this CIR. These compressed magnetic fields are located on the downstream flank of the SI and the analysis of their orientation should provide information on the orientation of the SI seen in situ, assuming that the magnetic field has a locally planar distribution. A minimum variance analysis (MVA) of the magnetic 
field fluctuations observed in that region was used to derive the distribution of the magnetic field (Sonnerup and Cahill, 1967). The minimum variance direction of the magnetic field variations measured between 5 UT on 21 July and 00 UT on 22 July 2007 is found (in RTN coordinates) to be $(-0.74,-0.36,-0.61)$. The eigenvalues for the minimum, intermediate and maximum variance were $e_{1}=3.26, e_{2}=18.65$ and $e_{3}=20.75$, respectively. The eigenvalue associated with the minimum variance direction is much smaller than for the maximum and intermediate directions, indicating that there exists a single vector orthogonal to most magnetic field lines during this interval (i.e., that the magnetic field is locally distributed in a plane). We can demonstrate this by plotting the magnetic field elevation angle as a function of the azimuth (Figure 10). A wavelike pattern is observed, which corresponds to a subset of all magnetic field directions possible inside this plane, that is, all directions satisfying the condition

$$
\mathbf{B} \cdot \mathbf{n}=\mathbf{0} .
$$

We overplot, in red, the expected relationship between azimuth and elevation angles for magnetic field lines distributed inside a plane, assuming that the minimum variance direction of the magnetic field is the normal to that plane. The SI plane is inclined at $54^{\circ}$ to the RT plane defined by the RTN coordinate system centred at STEREO-A. The intersection of the planar structure with the RT plane has an azimuth, $\phi_{\mathrm{SI}}$, of $118^{\circ}$ (i.e., it is $\sim 20^{\circ}$ more radial than the local Parker spiral field orientation or the MC axis). Previous studies of MCs entrained in interaction regions have found that the MC axis is often parallel to the stream interface. These in situ observations show that the density increase observed in the $\mathrm{HI}$ image (Figure 7) is a combination of solar wind and MC plasma compressed by the CIR, or compound stream. The central axis of this very small MC is nearly parallel to the SI and associated with a single large-scale increase of plasma ahead of the SI; therefore its topology cannot be distinguished from the CIR by using these HI observations alone.

Rouillard et al. (2008) noted that when plasma parcels are associated with CIRs, their appearance in $\mathrm{HI}$ images should depend on a combination of position relative to the Thomson sphere, the integration of density along the spiral arm and the amount of plasma gathered by kinematic steepening and compression (Burlaga and Barouch, 1976) at the coronal height of observation. Integration by $\mathrm{HI}-2 \mathrm{~B}$ of the light scattered by the coronal electrons compressed in the spiral arm of the CIR is enhanced when the tangent plane to that spiral includes HI- $2 \mathrm{~B}$.

The tilt of the CIR plasma density wave relative to the solar equator can be estimated from the STEREO-B HI images when the structure is observed edge-on, or, equivalently, when the tangent plane of the CIR wave includes the spacecraft. Such a scenario is drawn in Figure 3 and is discussed in more detail in the Appendix (Figure 12). An edge-on view would occur when the following condition is met:

$$
\alpha+\psi+\phi_{\mathrm{SI}}=\pi
$$

where $\phi_{\mathrm{SI}}$ is the angle of the tangent relative to the Sun-transient line. Provided that we have an estimate of $\phi_{\mathrm{SI}}$, we can calculate the elongation when the density wave is observed edge-on. For $\psi=16^{\circ}$ and $\phi_{\mathrm{SI}}=118^{\circ}$, this occurs when the plasma density wave passes an elongation of $\alpha=46^{\circ}$. A line of constant elongation, $\alpha=46^{\circ}$, is plotted in Figure 7 . The SI orientation of a CIR evolves during its radial propagation in rough accord with the orientation of the spiral arm (Lee, 2002). Parker spiral theory dictates that, between the radial distance of the wave seen in Figure 7 and the radial distance of STEREO-A, the local SI direction would vary by an azimuthal angle of $8^{\circ}$ at most. Hence the wave seen by HI$2 \mathrm{~B}$ in Figure 7 is nearly observed edge-on. Thus its inclination relative to the equator can 
be used to compare the inclination of the SI (located at the rear of the wave) relative to the Sun-STEREO-A line with the in situ observation of the SI orientation. A black/white dashed line plotted in Figure 7 shows the projection of a plane tilted by $54^{\circ}$ relative to the RT plane of STEREO-A at the elongation angle of the plasma density wave observed in Figure 7. This line orientation was calculated by assuming the plane is observed edgeon. The calculation of the orientation of this line in HI images is presented in full in the Appendix of this paper; it accounts for the orbital elements of STEREO-B, the pointing direction of the cameras, the distortion of the images and the projection of the line onto the angular coordinate system used in this paper. This line follows the orientation of the wave in $\mathrm{HI}-2 \mathrm{~B}$ very closely, suggesting that $\mathrm{HI}-2 \mathrm{~B}$ is indeed observing the radial and latitudinal distribution of the CIR plasma associated with the in situ observations.

This present study cannot reveal whether the orientation of the magnetic field observed in situ, or equivalently the orientation of the wave seen in HI, is related to the orientation of the streamer belt near the Sun (Lee, 2002) or whether it is related to the compression of plasma and magnetic fields by the CIR on the rear flank of the mini MC. The tentative association found here suggests that HI could be used to predict the orientation of the SI before it arrives at a point in the heliosphere.

\section{Concluding Remarks}

In this paper, a large-scale plasma element associated with the merging of a CIR and a smallscale transient (magnetic cloud) was observed from the Sun to STEREO-A. The aims of this paper were

- to disentangle the geometry of the CIR in HI images and to compare it with the in situ observations of this same CIR and

- to assess the suggestion made by Rouillard et al. (2008) and Sheeley et al. (2008a) that the parts of the CIRs seen in SECCHI difference images are primarily small-scale transients trapped inside CIRs.

The CIR observed by HI corresponded to a clear latitudinal and longitudinal distribution of compressed plasma. The stream interface, normally located at the rear of the density increase (Burlaga, 1974; Gosling et al., 1978), was located in the HI image by a ballistic back-mapping. The orientation of the SI derived from the HI images compared favourably to the SI orientation obtained by analysis of the compressed magnetic field inside the CIR. The directions of solar wind flow deflections in latitude around the SI were also correctly predicted from the $\mathrm{HI}$ images.

That section of the CIR observed by HI was predicted to pass over the STEREO-A spacecraft. A clear small-scale transient is observed in situ at STEREO-A, ahead of the SI passage and inside the plasma density increase. We suggest that the presence of this flux rope is the reason STEREO-B could observe the CIR so clearly in the HI difference images. The magnetic field lines of this CME were open and of the same polarity as the magnetic field lines of the equatorial coronal hole which forced the formation of the CIR. Moreover, the MC replaced locally the HCS connecting solar winds of different magnetic field polarity. Figure 11 shows a likely scenario that could have led to the magnetic field topology observed in situ. In this scenario, a small-scale flux rope formed and was lifted from the photosphere with a flux-rope axis nearly parallel to the solar equator (as seen in situ) (Figure 11a). The open magnetic field lines located on the boundary of coronal holes normally diverge over the photospheric areas dominated by closed field lines (Wang and Sheeley, 2003) and could 

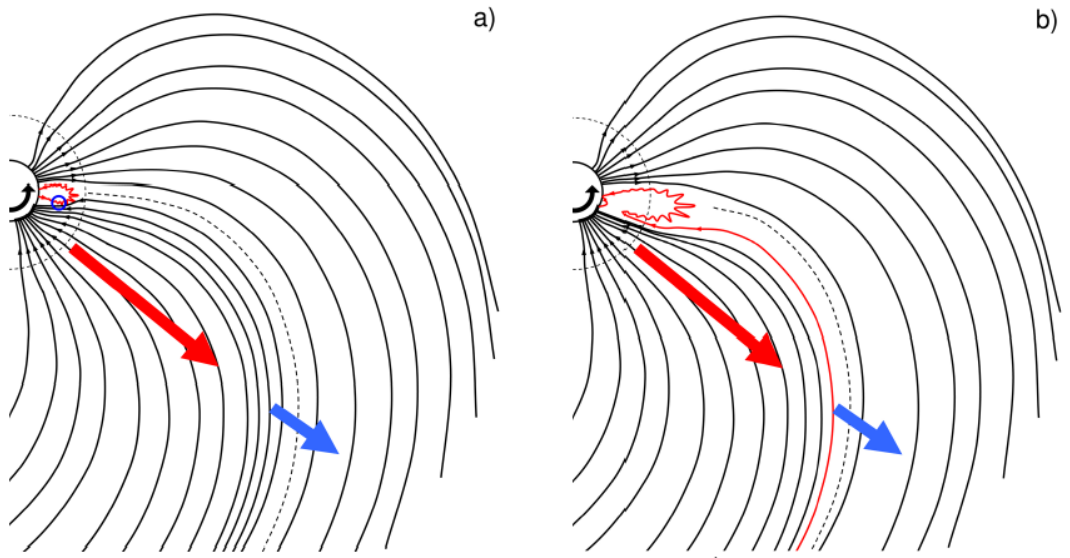

b)

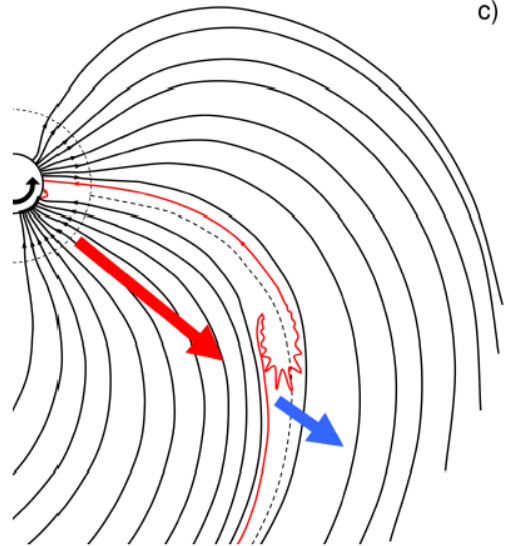

Figure 11 A view of the solar equatorial plane during the take-off of the small-scale transient. This scenario is inferred directly from the in situ observations of the solar wind made by STEREO-A and consists of a flux-rope emergence (a), reconnection of the flux rope with open field lines (b) and trapping of the small-scale transient inside a forming CIR (c). The HCS is plotted as a dotted black line in each panel. The location of the source region of the MC is fixed between each frame.

have obstructed the outward propagation of the CME before reconnection occurred. During its outward propagation, the inward footpoint of the CME has reconnected with the outward magnetic field lines of the coronal hole (Figure 11b). After reconnection the CME is connected to the interplanetary medium (red line) and progresses outwards in the slow solar wind. The fast solar wind emitted by the centre of the isolated coronal hole will interact with the slow solar wind and the slow MC higher up in the corona and compress both the slow solar wind and the MC (Figure 11c).

It is interesting to note that large equatorial coronal holes are more prone to force the formation of CIRs in the interplanetary medium because they often emit fast solar wind at the same latitude as the streamer belt. The proximity of the open magnetic field line footpoints located in these equatorial coronal holes with the closed magnetic field lines threading the photosphere near the neural line favour the reconnection of these open field lines with closed structures (flux ropes or closed loops of the magnetic carpet) leading to footpoint exchange. Small-scale transients trapped in CIRs can therefore be the result of footpoint exchange between open and closed field lines, flux ropes or completely disconnected magnetic field 
lines; each of these events can lead to significant variations in the plasma density of the solar wind, leading to longitudinal structure in CIRs higher in the corona. Figure 11 presents therefore only one possible scenario leading to small-scale transients in the slow solar wind. Wang et al. (2000), Zurbuchen et al. (2001) and Crooker et al. (2004) present reconnection events which could lead to other types of small-scale transients inside the slow solar wind. Kilpua et al. (2009) [this issue] present clear examples of small-scale transients present in the slow solar wind during Carrington Rotations 2054-2055. They investigated the origin of these transients and located the origin in the vicinity of coronal source surface sector boundaries. We will present in an upcoming paper a small-scale magnetic reconnection event that led to the presence of open and closed field lines inside a CIR during September 2007, observed simultaneously in images and in situ.

The entrainment of small-scale transients by CIRs will depend on the proximity in longitude of the transient to the CIR as well as the relative speed between the transient and the CIR. Hence HI difference images are expected to display a variability in brightness which will depend on the interaction between transients and CIRs; in other words plasma parcels can suddenly appear in HI difference images at large distances from the Sun when a CIR catches up with the transient.

Acknowledgements This work was funded by STFC (UK). The STEREO/SECCHI data are produced by a consortium of RAL (UK), NRL (USA), LMSAL (USA), GSFC (USA), MPS (Germany), CSL (Belgium), IOTA (France) and IAS (France). The PLASTIC and IMPACT data are produced by a consortium of the CESR (France), the University of New Hampshire and the University of California.

Open Access This article is distributed under the terms of the Creative Commons Attribution Noncommercial License which permits any noncommercial use, distribution, and reproduction in any medium, provided the original author(s) and source are credited.

\section{Appendix}

The tilt of the CIR plasma density wave relative to the solar equator can be estimated from $\mathrm{HI}$ images when the structure is observed edge-on or, equivalently, when the tangent of the CIR-associated spiral is directed along the STEREO-B line. Such a scenario is drawn in Figure 3. An edge-on view of the equatorial part of the wave (i.e., when $\beta=\psi$ ) occurs when the following condition is met:

$$
\alpha+\psi+\phi_{\mathrm{SI}}=\pi
$$

where $\phi_{\mathrm{SI}}$ is the angle of the tangent relative to the Sun - transient line. Provided we have an estimate of $\phi_{\mathrm{SI}}$, we can calculate the $\alpha$ angle corresponding to an edge-on view of that plane.

The radial-tangential-normal (RTN) axes at point $P$ are such that $\mathbf{R}$ is a unit vector pointing away from the Sun along the direction $S P$ and $\mathbf{T}$ is a unit vector pointing in the sense of solar rotation (i.e., is parallel to the solar equatorial plane, $x y$ ). The intersection of the SI plane with the RN plane is a line inclined at an angle $\delta$ with respect to the $\mathbf{R}$ direction (or $S P$ direction). $\delta$ can be related to the RTN components of the normal vector to the SI plane, $\mathbf{n}=(a, b, c)$, at point $P$ :

$$
\tan (\delta)=\frac{a}{c}
$$




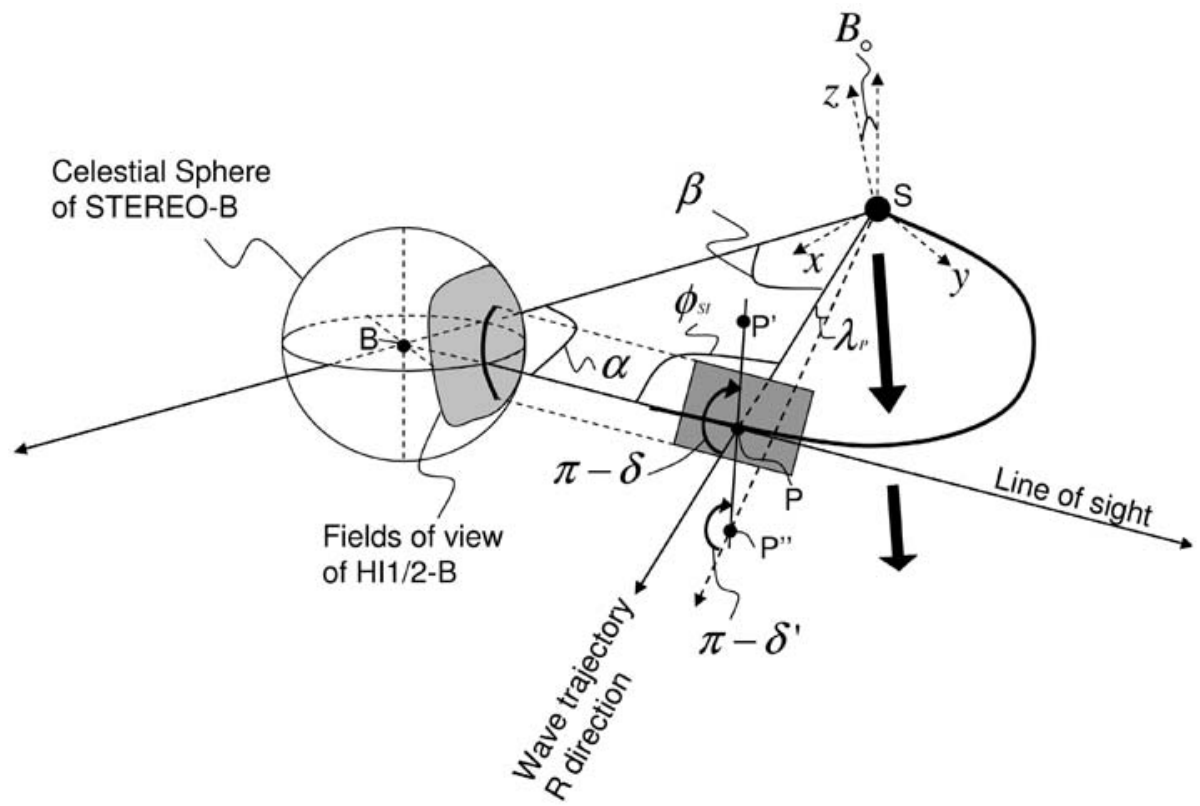

Figure 12 A schematic describing the outward propagation of a planar structure observed by SECCHI STEREO-B, showing the Sun (S), STEREO-B (B) and the outflowing planar plasma parcel (P). A heliocentric coordinate system is defined by the three axes $(x, y, z)$. The planar structure is shown on a Parker spiral field for a situation where STEREO-B observes the plane edge-on along the line of sight.

Let us define a heliocentric orthogonal coordinate system where the $x z$ plane contains the STEREO-B spacecraft and $x y$ is the solar equatorial plane. From the definition of RTN coordinates, the angle between the solar rotational axis and $\mathbf{N}$, angle $(\mathbf{N}, \mathbf{z})$, is the latitude of point $P, \lambda_{P}$. The equation of line $P P^{\prime}$ in 3-D space as a function of heliographic latitude $\left[\lambda=\operatorname{atan}\left(z / \sqrt{x^{2}+y^{2}}\right)\right]$ can be obtained by considering triangle $S P P^{\prime}$ :

$$
S P^{\prime}=\frac{S P \tan \left(\delta^{\prime}\right)}{\sin (\lambda)+\cos (\lambda) \tan \left(\delta^{\prime}\right)},
$$

where $\lambda$ is the heliographic latitude of point $P^{\prime}$ in Figure 12. $P^{\prime \prime}$ is defined as the point of intersection of line $P P^{\prime}$ with the equator. From the definition of RTN coordinates, we can calculate the angle, $\delta^{\prime}$, between line $S P$ and line $S P^{\prime \prime}$ :

$$
\delta^{\prime}=\delta-\lambda_{P}
$$

The distance $S P$ can be obtained from the elongation angle $\alpha$ of the wave when observed in the equatorial plane by STEREO-B such that $\beta=\psi$; using a rearranged form of Equation (1) this is

$$
S P=\frac{r_{I} \tan (\alpha)}{\sin (\beta)+\cos (\beta) \tan (\alpha)} .
$$

The line $P P^{\prime}$ can be expressed in a coordinate system unique to STEREO-B [i.e., projected onto the celestial sphere such as the (PA, $\alpha$ ) system used in this paper]. Then PA 
can be obtained from the heliocentric latitude $(\lambda)$ and longitude $(\phi)$ relative to STEREO-B (Sheeley et al., 2009):

$$
\mathrm{PA}=2 \pi-\operatorname{atan}\left[\frac{\cos (\lambda) \sin (\phi)}{-\cos (\lambda) \cos (\phi) \sin \left(B_{0}\right)+\sin (\lambda) \cos \left(B_{0}\right)}\right],
$$

where $B_{0}$ is the tilt of the solar rotational axis in the solar meridional plane that contains the STEREO-B spacecraft (i.e., the heliographic latitude of STEREO-B). We note that the quadrant in which the PA angle is evaluated by using Equation (9) must be considered with care. The $\beta$ angle variation along the line $P P^{\prime}$ can also be uniquely determined from the angles $\lambda$ and $\phi$ :

$$
\sin ^{2}(\beta)=\cos (\lambda)^{2} \sin (\phi)^{2}+\left[-\cos (\lambda) \cos (\phi) \sin \left(B_{0}\right)+\sin (\lambda) \cos \left(B_{0}\right)\right]^{2} .
$$

The $\alpha$ variation along the line $P P^{\prime}$ is then evaluated from the $\beta$ [Equation (10)] and the $S P^{\prime}$ [Equation (6)] variations using Equation (1) again:

$$
\tan (\alpha)=\frac{S P^{\prime} \sin (\beta)}{r I-S P^{\prime} \cos (\beta)}
$$

The $\alpha$ and PA variations are then determined on HI images from the helioprojective radial coordinate of each image pixel (Thomson, 2006).

\section{References}

Acuña, M.H., Curtis, D., Scheifele, J.L., Russell, C.T., Schroeder, P., Szabo, A., Luhmann, J.G.: 2008, Space Sci. Rev. 136, 203.

Burlaga, L.F.: 1974, J. Geophys. Res. 79, 3717.

Burlaga, L.F., Barouch, E.: 1976, Astrophys. J. 203, 257.

Burlaga, L.F., Behannon, K.H., Klein, L.W.: 1987, J. Geophys. Res. 92, 5725.

Burlaga, L.F., Hewish, A., Behannon, K.H.: 1991, J. Geophys. Res. 96, 21213.

Burlaga, L.F., Sittler, E., Mariani, F., Schwenn, R.: 1981, J. Geophys. Res. 86, 6673.

Burlaga, L.F., Goldstein, M.L., McDonald, F.B., Lazarus, A.J.: 1985, J. Geophys. Res. 90, 12027.

Burlaga, L.F., Berdichevsky, D., Gopalswamy, N., Lepping, R., Zurbuchen, T.: 2003, J. Geophys. Res. 108. doi:10.1029/2003JA010088.

Crooker, N.U., Huang, C.L., Lamassa, S.M., Larson, D.E., Kahler, S.W., Spence, H.E.: 2004, J. Geophys. Res. 109, CiteID A03107.

Eyles, C.J., Harrison, R.A., Davis, C.J., Waltham, N.R., Shaughnessy, B.M., Mapson-Menard, et al.: 2009, Solar Phys. 254, 387.

Fenrich, F.R., Luhmann, J.G.: 1998, Geophys. Res. Lett. 25, 2999.

Galvin, A.B., Kistler, L.M., Popecki, M.A., Farrugia, C.J., Simunac, K.D.C., Ellis, L., et al.: 2008, Space Sci. Rev. 136, 437.

Gazis, P.R.: 1996, Rev. Geophys. 34, 379.

Gosling, J.T., Asbridge, J.R., Bame, S.J., Feldman, W.C.: 1978, J. Geophys. Res. 83, 1401.

Howard, R.A., Moses, J.D., Vourlidas, A., Newmark, J.S., Socker, D.G., Plunkett, S.P., et al.: 2008a, Space Sci. Rev. 136, 67.

Howard, R.A., Thernisien, A., Vourlidas, A., Morrill, J., Mac-Neice, P., Luhman, J., Galvin, A.: 2008b, In: Meeting of the European Geophysical Union, Vienna.

Hudson, H.S., Bougeret, J.-L., Burkepile, J.: 2006, Space Sci. Rev. 123, 13.

Insley, J.E., Moore, V., Harrison, R.A.: 1995, Solar Phys. 160, 1.

Kilpua, E.K., Luhmann, J.G., Gosling, J., Li, Y., Elliott, H., Russell, C.T., et al.: 2009, Solar Phys., submitted. Lavraud, B., Thomsen, M.F., Borovsky, J.E., Denton, M.H., Pulkkinen, T.I.: 2006, J. Geophys. Res. 111, Issue A9, CiteID A09208.

Lee, M.A.: 2002, J. Geophys. Res. 105, 10491.

Luhmann, J.G., Li, Y., Arge, C.N., Gazis, P.R., Ulrich, R.: 2002, J. Geophys. Res. 107, CiteID 1154. 
Luhmann, J.G., Curtis, D.W., Schroeder, P., McCauley, J., Lin, R.P., Larson, D.E., et al.: 2008, Space Sci. Rev. 136, 117.

McComas, D.J., Bame, S.J., Barker, P., Feldman, W.C., Phillips, J.L., Riley, P., Griffee, J.W.: 1998, Space Sci. Rev. 86, 563.

Parker, E.N.: 1958, Astrophys. J. 128, 664.

Pizzo, V.J., Gosling, J.T.: 1994, Geophys. Res. Lett. 25, 1529.

Rees, A., Forsyth, R.J.: 2004, Geophys. Res. Lett. 31, CiteID L06804.

Rouillard, A.P., Davies, J.A., Forsyth, R.J., Rees, A., Davis, C.J., Harrison, R.A., et al.: 2008, Geophys. Res. Lett. 35, CiteID L10110.

Sauvaud, J.-A., Larson, D., Aoustin, C., Curtis, D., Médale, J.-L., Fedorov, A., Rouzaud, J., Luhmann, J.G., Moreau, T., Schröder, P., Louarn, P., Dandouras, I., Penou, E.: 2008, Space Sci. Rev. 136, 227.

Schwenn, R., Marsch, E.: 1990, In: Physics of the Inner Heliosphere 20, Springer, Berlin, 1.

Sheeley, N.R. Jr., Walters, J.H., Wang, Y.-M., Howard, R.A.: 1999, J. Geophys. Res. 104, 24739.

Sheeley, N.R. Jr., Herbst, A.D., Palatchi, C.A., Wang, Y.-M., Howard, R.A., Moses, J.D., et al.: 2008a, Astrophys. J. 674, L109.

Sheeley, N.R. Jr., Herbst, A.D., Palatchi, C.A., Wang, Y.-M., Howard, R.A., Moses, J.D., et al.: 2008b, Astrophys. J. 675, 853 .

Sheeley, N.R. Jr., Lee, D.D., Casto, K.P., Wang, Y.M., Rich, N.B.: 2009, Astrophys. J., in press.

Sonnerup, B.U.O., Cahill, L.J. Jr.: 1967, J. Geophys. Res. 72, 171.

Thomson, W.T.: 2006, Astron. Astrophys. 449, 791.

Vourlidas, A., Howard, R.A.: 2006, Astrophys. J. 642, 1216.

Wang, Y.M., Sheeley, N.R.: 2003, Astrophys. J. 599, 1404.

Wang, Y.M., Hawley, S.H., Sheeley, N.R.: 1996, Science 271, 464.

Wang, Y.M., Sheeley, N.R., Socker, D.G., Howard, R.A., Rich, N.B.: 2000, J. Geophys. Res. 105, 25133.

Zurbuchen, T.H., Hefti, S., Fisk, L.A., Gloeckler, G., Schwadron, N.A., Smith, C.W., Ness, N.F., Skoug, R.M., McComas, D.J., Burlaga, L.F.: 2001, J. Geophys. Res. 106, 16001. 